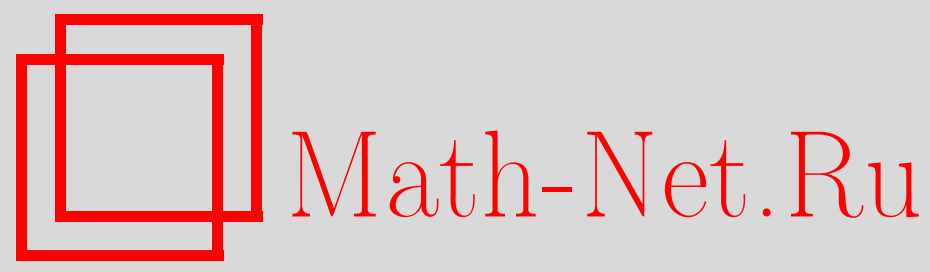

А. В. Бондаренко, А. В. Примак, Отрицательные результаты в формосохраняющем приближении высших порядков, Матем. заметки, 2004, том 76, выпуск 6, 812-823

DOI: https://doi.org/10.4213/mzm150

Использование Общероссийского математического портала Math-Net.Ru подразумевает, что вы прочитали и согласны с пользовательским соглашением http://www.mathnet.ru/rus/agreement

Параметры загрузки:

IP : 18.208 .226 .222

26 апреля 2023 г., 18:37:31

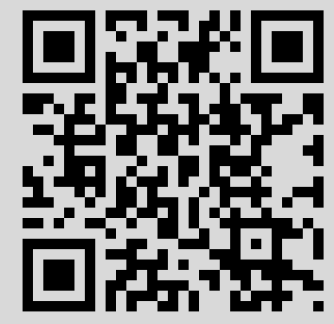




\title{
ОТРИЦАТЕЛЬНЫЕ РЕЗУЛЬТАТЫ В ФОРМОСОХРАНЯЮЩЕМ ПРИБЛИЖЕНИИ ВЫСШИХ ПОРЯДКОВ
}

\author{
А. В. Бондаренко, А.В. Примак
}

Доказано, что для $q$-выпуклого приближения многочленами при $q \geqslant 4$ не верны неравенства типа Джексона даже с константой, зависящей от приближаемой функции.

Библиографоия: 10 названий.

1. Введение. Приближая непрерывные на отрезке функции иногда важно сохранить определенные свойства функций. Например, монотонную функцию хотелось бы приблизить монотонньм многочленом, выпуклую - выпукльм.

Понятие выпуклость имеет естественное обобщение - $q$-вьпуклость. Обозначим чеpe3

$$
\left[x_{0}, x_{1}, \ldots, x_{q} ; f\right]:=\sum_{i=0}^{q} \frac{f\left(x_{i}\right)}{\prod_{j=0, j \neq i}^{q}\left(x_{i}-x_{j}\right)}
$$

разделенную разность функции $f$ в точках $x_{0}, \ldots, x_{q}$. Функция $f:[a, b] \rightarrow \mathbb{R}$ назьвается $q$-выпуклой на отрезке $[a, b]$ (будем писать $\left.f \in \Delta^{q}[a, b]\right)$, если $\left[x_{0}, x_{1}, \ldots, x_{q} ; f\right] \geqslant 0$ для всевозможных наборов $q+1$ различных точек $x_{0}, x_{1}, \ldots, x_{q} \in[a, b]$. В частности, если $f$, скажем, $q$ раз непрерьвно-дифференцируемая на $[a, b]$, то $f \in \Delta^{q}[a, b]$ означает, что $f^{(q)}(x) \geqslant 0, x \in[a, b]$. Легко видеть, что 1-вьпуклые и 2-выпуклые функции являются соответственно неубывающими и выпуклыми вниз функциями. Различные свойства $q$-выпуклых функций можно найти в [1], здесь мы лишш сформулируем следующую теорему.

Теорема. Пусть $q \geqslant 2 u f \in \Delta^{q}[a, b]$. Тогда для любого иелого неотрицательного $j \leqslant q-2$ существует $f^{(j)}$ на $(a, b)$ и является $(q-j)$-выпуклой. В частности, существует $f^{(q-2)}$, которая является выпуклой $и$, следовательно, абсолютно непрерывной на произвольном подотрезке интервала $(a, b)$.

Для функции $f \in C[-1,1]$ обозначим через

$$
E_{n}(f):=\inf _{P \in \mathscr{P}_{n}}\|f-P\|_{C[-1,1]}
$$

величину наилучшего равномерного приближения многочленами; здесь $\mathscr{P}_{n}-$ пространство алгебраических многочленов степени $\leqslant n$.

Работа обоих авторов выполненапри поддержке украино-французскогогранта “Днипро”, грант № M/262-2003. 
Аналогично, для $f \in C[-1,1]$ обозначим

$$
E_{n}^{(q)}(f):=\inf _{P \in \mathscr{P}_{n} \cap \Delta q[-1,1]}\|f-P\|_{C[-1,1]}
$$

величину наилучшего $q$-вьпуклого приближения многочленами.

Пусть $W_{\infty}^{r}-$ класс функций, имеющих абсолютно непрерьвную $(r-1)$-ю производную на $[-1,1]$, таких, что $\left\|f^{(r)}\right\|_{L_{\infty}}<\infty$. Для $f \in W_{\infty}^{r}$ хорошо известно следующее неравенство:

$$
E_{n}(f) \leqslant c(r) \frac{\left\|f^{(r)}\right\|_{L_{\infty}}}{n^{r}}, \quad n \geqslant r-1 .
$$

Здесь и далее, $n, r \in \mathbb{N}$ и через $c(\cdot)$ обозначены положительные постоянные, зависящие только от параметров, указанных в скобках.

Возникает естественный вопрос, верен ли аналог оценки (1) для $q$-вьпуклого приближения, а именно, верна ли для $f \in W_{\infty}^{r} \cap \Delta^{q}[-1,1]$ оценка

$$
E_{n}^{(q)}(f) \leqslant c(r, q) \frac{\left\|f^{(r)}\right\|_{L_{\infty}}}{n^{r}}, \quad n \geqslant M .
$$

Справедливость (2) с $M=r-1$ для $r=1, q=1$ была установлена Лоренщем и Целлером [2], для $r=2, q=1$ - Лоренцем [3], для $r>2, q=1$ - Девором [4], для $r=1, q>1$ Битсоном [5], для $r=2, q>1$ - Шведовьм [6], для $r>2, q=2$ - Манией и Шевчуком [7, c. 148]. Позитивный ответ для случая $r=3, q=3$ получен первьм автором в [8]. Для $q=3, r \geqslant 4$ вопрос остается открытым.

При $q \geqslant 4$ даже неравенство (2) оказывается не верным ни при каком $r>2$ c $M=$ $M(r)$. Это следует из результатов Коновалова и Левиатана [9] о формосохраняющих поперечниках. Точнее, вместо ожидаемой скорости приближения $n^{-r}$ при $q \geqslant 4$ получен порядок формосохраняющего поперечника $n^{-r+q-3}$, т.е. теряется как минимум один порядок аппроксимации.

В настоящей работе доказано, что при $q \geqslant 4$ и $r>2$ неравенство (2) не верно даже с $M=M(f)$ и $c=c(f)$. Случай $r<q-1$ рассмотрен в теореме 2 , а случай $r \geqslant q-1-$ в теореме 3 .

ЗАМЕчАнИЕ 1. В формосохраняющем приближении известны результаты о том, что соответствующая оценка не верна с $M=M(r)$, но верна с $M=M(f)$. Например, согласно Шведову [6] для любых $A>0, n \in \mathbb{N}$ существует $f=f_{A, n} \in \Delta^{1}[-1,1]$ такая, что

$$
E_{n}^{(1)}(f)>A \omega_{3}\left(f, \frac{1}{n}\right)
$$

где $\omega_{3}(f, t)$ - модуль непрерьвности порядка 3 ; с другой стороны, Левиатан и Шевчук [10] доказали неравенство

$$
E_{n}^{(1)}(f) \leqslant c \omega_{3}\left(f, \frac{1}{n}\right), \quad n>M(f),
$$

в котором $c$ - абсолютная постоянная.

Для доказательства теорем важным оказьвается переход от выпуклого приближения функции $|x|$ к 3 -выпуклому приближению $\frac{1}{2} x^{2} \operatorname{sign} x$, когда удается доказать существование достаточно “длинного" интервала с соответствующим отклонением; это результат теоремы 1. 
Теорема 1. Пусть $F-3$-выпуклая на $[-1,1]$ функиия,

$$
d:=\left\|F^{\prime}(x)-|x|\right\|_{C[-1 / 2,1 / 2]} .
$$

Тогда существует отрезок $I \subset[-1,1]$ длинь $|I| \geqslant 1 / 32$ такой, что

$$
\left|F(x)-\frac{x^{2} \operatorname{sign} x}{2}\right| \geqslant \min \left\{\frac{1}{3 \cdot 2^{5}} d, \frac{1}{2^{10}} d^{2}\right\} \text {. }
$$

ЗАмЕчАниЕ 2. Из теоремы 1 заменой переменных легко получить, что для 3-выпуклой на $[-1,1]$ функции $F$ при

$$
d:=\left\|F^{\prime}(x)-x_{+}\right\|_{C[-1 / 2,1 / 2]}
$$

(здесь и далее, $\left.x_{+}=\max \{x ; 0\}, x_{-}=\min \{x ; 0\}\right)$ существует отрезок $I \subset[-1,1]$, $|I| \geqslant 1 / 32$, такой, что при всех $x \in I$

$$
\left|F(x)-\frac{x_{+}^{2}}{2}\right| \geqslant \min \left\{\frac{1}{3 \cdot 2^{5}} d, \frac{1}{2^{10}} d^{2}\right\} .
$$

Teоpema 2. Пусть $q>3$. Тогдa

$$
E_{n}^{(q)}\left(x_{+}^{q-1}\right) \geqslant \frac{c(q)}{n^{2}}
$$

ТЕОРема 3. Для любых $r, q \in \mathbb{N}, r \geqslant q-1, q \geqslant 4$, и последовательности $\left\{\alpha_{n}\right\}_{n=1}^{\infty}$, $\alpha_{n} \geqslant 0, \lim _{n \rightarrow \infty} \alpha_{n}=+\infty$, существует функиия $f=f_{r, q} \in C^{(r)}[-1,1] \cap \Delta^{q}[-1,1]$ maкая, чmo

$$
\varlimsup_{n \rightarrow \infty} \alpha_{n} E_{n}^{(q)}(f) n^{r-q+3}=+\infty .
$$

Для наглядности объединим известные результаты, а также теоремы 2 и 3 в следующей таблице:

$$
\begin{array}{ccccccc}
r & \vdots & \vdots & \vdots & \vdots & \vdots & . \\
5 & + & + & ? & - & - & \cdots \\
4 & + & + & ? & - & - & \cdots \\
3 & + & + & + & - & - & \cdots \\
2 & + & + & + & + & + & \cdots \\
1 & + & + & + & + & + & \cdots \\
1 & 2 & 3 & 4 & 5 & q
\end{array}
$$

Здесь “+” означает, что (2) верно, причем с $M=r-1$; “-” означает, что (2) не верно даже с $N=N(f)$ и $c=c(f)$.

ЗАМЕчАНИЕ 3 . Несложной модификацией конструкции $f$ в доказательстве теоремы 3 можно получить тот же результат для приближения кусочно-многочленными функциями с равноудаленными узлами.

Следуюшее замечание является непосредственным следствием теоремы 3 и классических результатов о приближении без ограничений. 
ЗАмечАниЕ 4 . Пусть $q \geqslant 4, \alpha>2$. Тогда существует функция $f=f_{\alpha, q} \in \Delta^{q}[-1,1]$ такая, что

$$
E_{n}(f) \leqslant \frac{1}{n^{\alpha}}, \quad n=1,2, \ldots,
$$

но в то же время для некоторого $\delta>0$

$$
E_{n}^{(q)}(f) \geqslant \frac{c(\alpha, \delta, q)}{n^{\alpha-\delta}}, \quad n=1,2, \ldots
$$

ЗАмЕчаниЕ 5 . Теорема 1 влечет, что для 3 -выпуклого приближения в интегральной метрике $L_{p}[-1,1], 1 \leqslant p<\infty$, при $f \in \Delta^{3}[-1,1]$ даже оценка

$$
E_{n}^{(3)}(f)_{p} \leqslant c \omega_{3}\left(f, \frac{1}{n}\right)_{p}
$$

не является верной с $c=c(f)$, где $E_{n}^{(3)}(f)_{p}$ и $\omega_{3}(f, 1 / n)_{p}$ - соответствующие аналоги величины наилучшего приближения и модуля непрерьвности в $L_{p}$. Для того чтобы показать это, достаточно взять $f(x)=x_{+}^{2}, x \in[-1,1]$.

В п. 2 доказьваются вспомогательные леммы, точнее, леммы 1-6 для доказательства теорем 1, 2 и лемма 7 (используюшая теорему 2) для доказательства теоремы 3. Сами доказательства теорем содержатся в п. 3. Для сокращения обозначений в формулировках и доказательствах леммы 7 и теоремы 3 константы $c, c_{0}, c_{1}, \ldots$ зависят от $r$ и $q$. Теоремы 1,2 доказаны А. В. Бондаренко, теорема 3 - А. В. Примаком.

\section{2. Вспомогательные леммы.}

Лемма 1. Пусть $g$ - выпуклая на $[a, b]$ функиия, $x^{*}-$ точка на $[a, b]$ такая, что $-g\left(x^{*}\right)=: h>0$. Тогда существует интервал $I \subset[a, b]$ длины не менее чем $(b-a) / 8$ такой, что $|g(x)| \geqslant h / 2$ при всех $x \in I$.

ДокАЗАТЕЛЬСТво. Неограничивая общности, можно считать, что $x^{*} \in[(a+b) / 2, b]$. Пусть

$$
x_{1}:=\max \left\{a,\left\{x: g(x) \geqslant 0, x \in\left[a, x^{*}\right]\right\}\right\},
$$

И

$$
l(x):=\frac{h}{x_{1}-x^{*}}\left(x-x^{*}\right)-h .
$$

Заметим, что $l\left(x^{*}\right)=-h$; кроме того, $l\left(x_{1}\right)=0, x_{1}<x^{*}$, поэтому $l$ убывает.

Из выпуклости $g$ следует, что

$$
\begin{aligned}
& g(x) \geqslant l(x), \quad x \in\left(a, x_{1}\right), \\
& g(x) \leqslant l(x), \quad x \in\left[x_{1}, x^{*}\right] .
\end{aligned}
$$

Докажем, что выполняется хотя бы одно из неравенств

$$
\begin{aligned}
& l\left(\frac{7 a+b}{8}\right)=\frac{h}{x^{*}-x_{1}}\left(x^{*}-\frac{7 a+b}{8}\right)-h \geqslant \frac{h}{2}, \\
& l\left(x^{*}-\frac{b-a}{8}\right)=\frac{h}{x^{*}-x_{1}}\left(\frac{b-a}{8}\right)-h \leqslant-\frac{h}{2} .
\end{aligned}
$$


Тогда, учитьвая (4) и (5), получим, что один из отрезков $[a,(7 a+b) / 8]$ или $\left[x^{*}-\right.$ $\left.(b-a) / 8, x^{*}\right]$ является искомым. Предположим, что $(7)$ не выполняется. Тогда

$$
\frac{h}{x^{*}-x_{1}}>\frac{4 h}{b-a} \text {. }
$$

Из этого неравенства и отрицания к (6) следует, что $x^{*}<(a+b) / 2$; противоречие. Лемма 1 доказана.

ЛЕмма 2. Пусть $l(x)$ - линейная функиия такая, что для некоторого $x^{*} \in$ $[-1 / 2,1 / 2]$

$$
l\left(x^{*}\right)-\left|x^{*}\right|=: d>0
$$

$l(-1) \leqslant 1$ ul(1) $\leqslant 1$. Tогдa

$$
\int_{-1 / 4}^{1 / 4}(l(x)-|x|)+d x \geqslant \frac{1}{4} d^{2} .
$$

ДокАЗАТЕЛЬСТво. Из условий леммы следует существование $x_{1}, x_{2} \in(0,1]$ таких, что $l\left(-x_{1}\right)=x_{1}$ и $l\left(x_{2}\right)=x_{2}$. Тогда

$$
l(x)=\frac{\left(x_{2}-x_{1}\right) x+2 x_{1} x_{2}}{x_{1}+x_{2}}, \quad x \in \mathbb{R} .
$$

Легко видеть, что

$$
\int_{0}^{\min \left\{x_{2}, 1 / 4\right\}}(l(x)-x) d x= \begin{cases}\frac{1}{2} x_{2} l(0), & x_{2}<\frac{1}{4} \\ \frac{1}{4} l(0)\left(1-\frac{1}{8 x_{2}}\right), & x_{2} \geqslant \frac{1}{4}\end{cases}
$$

В любом случае

$$
\int_{0}^{\min \left\{x_{2}, 1 / 4\right\}}(l(x)-x) d x \geqslant \frac{1}{8} x_{2} l(0) .
$$

Аналогично

$$
\int_{\max \left\{-x_{1},-1 / 4\right\}}^{0}(l(x)+x) d x \geqslant \frac{1}{8} x_{1} l(0) .
$$

Имеем

$$
\begin{aligned}
\int_{-1 / 4}^{1 / 4}(l(x)-|x|)+d x & =\int_{\max \left\{-x_{1},-1 / 4\right\}}^{0}(l(x)+x) d x+\int_{0}^{\min \left\{x_{2}, 1 / 4\right\}}(l(x)-x) d x \\
& \geqslant \frac{1}{8}\left(x_{1}+x_{2}\right) l(0) \geqslant \frac{1}{4}(l(0))^{2} \geqslant \frac{1}{4}\left(l\left(x^{*}\right)-\left|x^{*}\right|\right)^{2}=\frac{1}{4} d^{2} .
\end{aligned}
$$

Лемма 2 доказана. 
Лемма 3. Пусть $l(x)$ - линейная функция такая, что для некоторого $x^{*} \in$ $[-1 / 2,1 / 2]$

$$
l\left(x^{*}\right)-\left|x^{*}\right|=: d>0
$$

и выполняется хотя бы одно из условий: $l(-1)>1$ или $l(1)>1$. Тогда существует отрезок $I \subset[-1,1],|I| \geqslant 1 / 4$, такой, что при всех $x \in I$

$$
l(x)-|x| \geqslant \frac{d}{6}
$$

ДокАЗАТЕЛЬСТво. Не ограничивая общности, можно считать, что $l(1)>1$; возьмем $I:=[1 / 2,3 / 4]$. Тогда $l_{1}(x):=l(x)-x$-линейная функция; кроме того, $l_{1}(1)>0$. Поскольку $l_{1}\left(x^{*}\right) \geqslant d$ и $x^{*} \geqslant-1 / 2$, имеем $l_{1}(1 / 2) \geqslant d / 3$ и $l_{1}(3 / 4) \geqslant d / 6$, поэтому $l_{1}(x)>d / 6$ при всех $x \in I$. Лемма 3 доказана.

Лемма 4. Пусть функиия $G \in C^{(1)}[a, b]$ удовлетворяет условию $\left|G^{\prime}(x)\right| \geqslant h n р и$ всех $x \in[a, b]$. Тогда существует отрезок $I \subset[a, b],|I| \geqslant(b-a) / 4$, такой, что при всех $x \in I$

$$
|G(x)| \geqslant \frac{h(b-a)}{4} \text {. }
$$

ДокАЗАТЕЛЬСТво. Не ограничивая обшности, можно считать, что $G^{\prime}(x) \geqslant h$ при всех $x \in[a, b]$. Пусть $G((a+b) / 2) \geqslant 0$. Положим $I:=[(a+3 b) / 4, b]($ для $G((a+b) / 2)<0$ возьмем $I:=[a,(3 a+b) / 4])$. Для произвольного $x \in I$ имеем

$$
\begin{aligned}
G(x) & \geqslant G\left(\frac{a+3 b}{4}\right) \geqslant G\left(\frac{a+3 b}{4}\right)-G\left(\frac{a+b}{2}\right) \\
& =\int_{(a+b) / 2}^{(a+3 b) / 4} G^{\prime}(x) d x \geqslant \int_{(a+b) / 2}^{(a+3 b) / 4} h d x=\frac{h(b-a)}{4} .
\end{aligned}
$$

Лемма 4 доказана.

Прямым следствием леммы 4 является лемма 5 .

Лемма 5. Пусть $s \in \mathbb{N}, G \in C^{(s)}[a, b]$ удовлетворяет условию $\left|G^{(s)}(x)\right| \geqslant h$ при всех $x \in[a, b]$. Тогда существует отрезок $I \subset[a, b],|I| \geqslant(b-a) / 4^{s}$, такой, ито при всех $x \in I$

$$
|G(x)| \geqslant \frac{h(b-a)^{s}}{2^{s^{2}+s}} .
$$

Лемма 6. Пусть $\partial л_{я} G \in C^{(1)}[-1 / 2,1 / 2]$

$$
\int_{-1 / 4}^{1 / 4}\left(G^{\prime}(x)\right)_{+} d x \geqslant A>0 \quad u \quad-\int_{-1 / 2}^{1 / 2}\left(G^{\prime}(x)\right)_{-} d x \leqslant \frac{A}{4} .
$$

Тогда отрезок $I=[-1 / 2,-1 / 4]$ или $I=[1 / 4,1 / 2]$ удовлетворяет следующему условию: оченка $|G(x)| \geqslant A / 4$ выполнена при всех $x \in I$. 
ДоКАЗАТЕЛЬСТво. Положим

$$
A_{*}:=\max _{[-1 / 2,-1 / 4]} G(x), \quad A^{*}:=\min _{[1 / 4,1 / 2]} G(x) .
$$

Тогда из условий леммы имеем

$$
A^{*}-A_{*} \geqslant \frac{3}{4} A
$$

Если

$$
A^{*} \geqslant \frac{1}{4} A
$$

то отрезок $[1 / 4,1 / 2]$ искомьй. Если же

$$
A^{*}<\frac{1}{4} A,
$$

то

$$
A_{*} \leqslant A^{*}-\frac{3}{4} A<-\frac{1}{2} A,
$$

и искомым будет отрезок $[-1 / 2,-1 / 4]$. Лемма 6 доказана.

Лемма 7. Для каждого $h \in(0,1), q, r$ таких, что $r \geqslant q-1, q \geqslant 4$, существуют $c_{1}, c_{2}>0 u f_{h}=f_{h, r, q} \in C^{(r)}[-2,1] \cap \Delta^{q}[-2,1]$, удовлетворяющая следующим условиям:

A1) $\left.f_{h}\right|_{[-2,0]}=0$;

А2) выполнена оченка

$$
\left\|f_{h}^{(i)}\right\|_{C[-2,1]} \leqslant \begin{cases}\frac{c}{h^{i-q+1}}, & i=q-1, \ldots, r, \\ 1, & i=0, \ldots, q-2 ;\end{cases}
$$

A3) если $h \leqslant c_{2} l^{(q-1) / 2} / n, l \in(0,1), \theta \in(0, l / 3), m o$

$$
E_{n}^{(q)}\left(f_{h}(x-\theta) ;[-l, l]\right) \geqslant \frac{c_{1} l^{q-1}}{n^{2}},
$$

где $E_{n}^{(q)}\left(f_{h}(x-\theta) ;[-l, l]\right)$ - величина наилучшего q-выпуклого приближения функиии $f_{h}(x-\theta)$ многочленами степени $\leqslant n$ на отрезке $[-l, l]$.

ДоКАЗАТЕЛЬСТво. Для Удобства возьмем $m:=r-q+1 \geqslant 0$. Положим

$$
f_{h}(x):=\int_{-2}^{x} \int_{-2}^{x_{1}} \cdots \int_{-2}^{x_{q-2}} s\left(x_{q-1}\right) d x_{q-1} \cdots d x_{1},
$$

где $s$ определена как

$$
s(x):= \begin{cases}0, & x \in[-2,0], \\ \frac{\int_{0}^{x} u^{m}(h-u)^{m} d u}{\int_{0}^{h} u^{m}(h-u)^{m} d u}, & x \in[0, h], \\ 1, & x \in[h, 1] .\end{cases}
$$


Легко видеть, что $s \in C^{(m)}[-2,1] \cap \Delta^{1}[-2,1]$, поэтому $f_{h} \in C^{(r)}[-2,1] \cap \Delta^{q}[-2,1]$. Также ясно, что

$$
f_{h}^{(q-1-j)}(x) \leqslant \frac{1}{j !} x^{j} \leqslant 1, \quad x \in[0,1], \quad j=0, \ldots, q-1 .
$$

Из неравенства Маркова для многочленов следует

$$
\left\|s^{(i)}\right\|_{C[-2,1]} \leqslant c(i) \frac{1}{h^{i}}, \quad i=0, \ldots, m,
$$

что влечет А2). Положим

$$
\lambda:=h-\int_{0}^{h} s(u) d u
$$

при этом $\lambda \in(0, h)$ и

$$
f_{h}^{(q-2)}(x)=(x-\lambda)_{+}, \quad x \in[-2,0] \cup[h, 1] .
$$

Следовательно, так как $0 \leqslant f_{h}^{(q-1)}(x) \leqslant 1, x \in[-2,1]$, то

$$
\left\|f_{h}^{(q-3)}(x)-\frac{1}{2}(x-\lambda)_{+}^{2}\right\|_{C[-2,1]} \leqslant h^{2} .
$$

Последовательно интегрируя, получим

$$
\left\|f_{h}(x)-\frac{1}{(q-1) !}(x-\lambda)_{+}^{q-1}\right\|_{C[-2,1]} \leqslant h^{2},
$$

что с учетом положительности $\theta$ влечет

$$
\left\|f_{h}(x-\theta)-\frac{1}{(q-1) !}(x-\theta-\lambda)_{+}^{q-1}\right\|_{C[-1,1]} \leqslant h^{2} .
$$

Из теоремы 2 следует оценка

$$
E_{n}^{(q)}\left(\frac{1}{(q-1) !}(x-\theta-\lambda)_{+}^{q-1} ;[-1,1]\right) \geqslant \frac{c_{3}}{n^{2}},
$$

которая после переноса на $[-l, l]$ примет вид

$$
E_{n}^{(q)}\left(\frac{1}{(q-1) !}(x-\theta-\lambda)_{+}^{q-1} ;[-l, l]\right) \geqslant \frac{c_{0} l^{q-1}}{n^{2}} .
$$

Причем в последнем неравенстве были использованы оценки $\lambda \leqslant l / 3$ и $\theta \leqslant l / 3$, которые следуют из определения $\lambda$ и условия А 3 ) при $c_{2} \leqslant 1 / 3$.

Если $c_{2} \leqslant \sqrt{c_{0} / 2}$ и $h \leqslant c_{2} l^{(q-1) / 2} / n$, то

Отсюда, из (8) и (9) получим

$$
\frac{c_{0} l^{q-1}}{2 n^{2}} \geqslant h^{2} \text {. }
$$

$$
\begin{aligned}
E_{n}^{(q)}\left(f_{h}(x-\theta) ;[-l, l]\right) \geqslant & E_{n}^{(q)}\left(\frac{1}{(q-1) !}(x-\theta-\lambda)_{+}^{q-1} ;[-l, l]\right) \\
& -\left\|f_{h}(x-\theta)-\frac{1}{(q-1) !}(x-\theta-\lambda)_{+}^{q-1}\right\|_{C[-1,1]} \\
& \geqslant \frac{c_{0} l^{q-1}}{n^{2}}-h^{2} \geqslant \frac{c_{0} l^{q-1}}{2 n^{2}},
\end{aligned}
$$

итак, А3) выполнено с $c_{1}:=c_{0} / 2$ и $c_{2}:=\min \left\{1 / 3 ; \sqrt{c_{0} / 2}\right\}$. Лемма доказана. 


\section{3. Доказательства теорем.}

ДоКАЗАТЕЛЬСТВО ТЕОРЕМЫ 1. Положим

$$
f(x):=F^{\prime}(x), \quad h_{1}:=-\min _{[-1 / 2,1 / 2]}(f(x)-|x|) .
$$

Точка, где этот минимум достигается, принадлежит $[-1,0]$ или $[0,1]$. Если $h_{1}>0$, то для этого отрезка и функции $g(x)=f(x)-|x|$ применим лемму 1 . К полученному отрезку $I$ такому, что $|I| \geqslant 1 / 8$ и при всех $x \in I|g(x)| \geqslant h_{1} / 2$, применим лемму 4 , где

$$
G(x):=F(x)-\frac{x^{2} \operatorname{sign} x}{2} .
$$

Получим, что существует отрезок длины не менее чем $1 / 32$, для каждой точки $x$ которого вьполняется неравенство

$$
\left|F(x)-\frac{x^{2} \operatorname{sign} x}{2}\right| \geqslant \frac{h_{1}}{2^{6}} .
$$

Поэтому можно считать, что

$$
h_{1} \leqslant \frac{1}{2^{4}} d^{2}
$$

(иначе выполняется (3)). Таким образом, при всех $x \in[-1 / 2,1 / 2]$

поэтому

$$
f(x) \geqslant|x|-\frac{1}{2^{4}} d^{2},
$$

$$
-\int_{-1 / 2}^{1 / 2}(f(x)-|x|)-d x \leqslant \frac{1}{2^{4}} d^{2} .
$$

По условию существует $x^{*} \in[-1 / 2,1 / 2]$ такая, что $\left|f\left(x^{*}\right)-\right| x^{*}||=d$; кроме того, из (10) следует, что $f\left(x^{*}\right)-\left|x^{*}\right|=d$. Если $d=0$, то (3) очевидно. Пусть $d>0$. Поскольку $f$ вьпукла, то существует линейная функция $l$ такая, что $l\left(x^{*}\right)=f\left(x^{*}\right)$ и при всех $x \in[-1,1] l(x) \leqslant f(x)$. Если $l(-1)>1$ или $l(1)>1$, то применим лемму 3 для функции $l(x)$. Для полученного отрезка и функции

$$
G(x)=F(x)-\frac{x^{2} \operatorname{sign} x}{2}
$$

применим лемму 4 , в результате получим отрезок $I \subset[-1,1],|I| \geqslant 1 / 16$, такой, что при вcex $x \in I$

$$
\left|F(x)-\frac{x^{2} \operatorname{sign} x}{2}\right| \geqslant \frac{1}{3 \cdot 2^{5}} d,
$$

что и нужно было доказать. Имеем $l(-1) \leqslant 1$ и $l(1) \leqslant 1$. Из леммы 2 следует, что

$$
\int_{-1 / 4}^{1 / 4}(f(x)-|x|)+d x \geqslant \frac{1}{4} d^{2}=: A .
$$

Теперь, учитывая (11), применим лемму 6 для функции

$$
G(x)=F(x)-\frac{x^{2} \operatorname{sign} x}{2} ;
$$

как результат, получим отрезок $I,|I| \geqslant 1 / 4$, такой, что при всех $x \in I$

$$
|G(x)| \geqslant \frac{1}{2^{4}} d^{2} .
$$

Теорема 1 доказана. 
ДокаЗАТЕЛЬСтво теоРемы 2. Пусть $Q \in \mathscr{P}_{n} \cap \Delta^{q}$. Тогда несложным применением неравенства Бернштейна для алгебраических многочленов можно получить, что

$$
\left\|(q-1) !^{-1} Q^{(q-2)}(x)-x_{+}\right\|_{C[-1 / 2,1 / 2]}>\frac{1}{2^{10} n}
$$

кроме того, как легко заметить, функция $(q-1) !^{-1} Q^{(q-2)}$ выпукла. Поэтому, замечание 2 обеспечивает существование отрезка $I \subset[-1,1],|I| \geqslant 1 / 32$, такого, что

$$
\left|(q-1) !^{-1} Q^{(q-3)}(x)-\frac{x_{+}^{2}}{2}\right| \geqslant \frac{1}{2^{30} n^{2}} .
$$

Применим лемму 5 при $s=q-3$ для функции

$$
G(x)=\frac{Q(x)}{(q-1) !}-\frac{x_{+}^{(q-1)}}{(q-1) !} .
$$

Получим

$$
\|G\|_{[-1,1]} \geqslant \frac{1}{2^{30} n^{2}} \cdot \frac{1}{32^{q-3}} \cdot \frac{1}{2^{(q-3)^{2}+q-3}} .
$$

Отсюда

$$
\left\|Q(x)-x_{+}^{q-1}\right\|_{C[-1,1]} \geqslant \frac{(q-1) !}{2^{q^{2}+21}} \cdot \frac{1}{n^{2}}=: \frac{c(q)}{n^{2}} .
$$

Поскольку многочлен $Q$ был произвольным, получим

$$
E_{n}^{(q)}\left(x_{+}^{q-1}\right) \geqslant \frac{c(q)}{n^{2}} .
$$

Теорема 2 доказана.

ДоКАЗАТЕЛЬСТВо ТЕОРЕМЫ 3 . Для Удобства обозначим $m:=r-q+1 \geqslant 0$. Легко видеть, что можно выбрать строго возрастающую последовательность $\left\{n_{k}\right\}_{n=1}^{\infty}$ натуральных чисел такую, что

$$
\begin{gathered}
\lim _{k \rightarrow \infty} \alpha_{n_{k}} \frac{1}{n_{k-1}^{(m+1)(q-1)+2}}=+\infty, \\
\sum_{k=s+1}^{\infty} \frac{1}{n_{k}^{m} n_{k-1}^{m(q-1)+2}} \leqslant 2 \frac{1}{n_{s+1}^{m} n_{s}^{m(q-1)+2}}, \quad s \in \mathbb{N}, \\
\frac{c_{1}}{n_{s}^{m+2} n_{s-1}^{(m+1)(q-1)+2}} \geqslant 4 \frac{1}{n_{s+1}^{m} n_{s}^{m(q-1)+2}}, \quad s \geqslant 2, \\
n_{s+1}>3 n_{s}, \quad s \in \mathbb{N} .
\end{gathered}
$$

Возьмем достаточно быстро растущую последовательность $\left\{n_{k}\right\}$. Определим искомую функцию $f$ :

$$
f(x):=\sum_{k=2}^{\infty} \beta_{k} f_{h_{k}}\left(x-x_{k}\right),
$$


где $f_{h_{k}}-$ функции из леммы 7 ,

$$
h_{k}:=\frac{1}{c_{2} n_{k} n_{k-1}^{q-1}}, \quad \beta_{k}:=\frac{1}{n_{k+1}^{m} n_{k}^{m(q-1)+2}}, \quad x_{k}:=\frac{1}{n_{k}} .
$$

Заметим, что при этом (13) и (14) примут вид

$$
\begin{aligned}
& \sum_{k=s+1}^{\infty} \beta_{k} \leqslant 2 \beta_{s+1}, \quad s \in \mathbb{N}, \\
& \frac{c_{1} \beta_{s} x_{s-1}^{q-1}}{n_{s}^{2}} \geqslant 4 \beta_{s+1}, \quad s \geqslant 2 .
\end{aligned}
$$

Покажем, что $f$ определена корректно и $f \in C^{(r)}[-1,1]$. Для $j=0, \ldots, r$, учитывая А2), имеем

$$
\begin{aligned}
\sum_{k=2}^{\infty} \beta_{k}\left\|f_{h_{k}}^{(j)}\right\|_{C[-2,1]} & \leqslant \sum_{k=2}^{\infty} \frac{1}{n_{k}^{m} n_{k-1}^{m(q-1)+2}} \max \left\{1 ; c\left(c_{2}^{-1} n_{k} n_{k-1}^{q-1}\right)^{j-q+1}\right\} \\
& \leqslant \sum_{k=2}^{\infty} \frac{1}{n_{k}^{m} n_{k-1}^{m(q-1)+2}} c\left(c_{2}^{-1} n_{k} n_{k-1}^{q-1}\right)^{m} \\
& =\sum_{k=2}^{\infty} \frac{c}{n_{k-1}^{2} c_{2}^{m}}<\frac{c}{c_{2}^{m}} \sum_{n=1}^{\infty} \frac{1}{n^{2}}<+\infty
\end{aligned}
$$

Для каждого $k \geqslant 2$

$$
\beta_{k} f_{h_{k}}\left(x-x_{k}\right) \in \Delta^{q}[-1,1]
$$

следовательно, $f \in \Delta^{q}[-1,1]$ как равномерньй предел $q$-вьпуклых функций. Теперь будем оценивать $E_{n_{s}}^{(q)}(f ;[-1,1])$ снизу, $s \geqslant 2$. Заметим, что ввиду А1) и определения $f$

$$
f(x)=\sum_{k=s}^{\infty} \beta_{k} f_{h_{k}}\left(x-x_{k}\right), \quad x \in\left[-1, x_{s-1}\right] .
$$

Учитьвая А2) и (16), находим

$$
\left\|\sum_{k=s+1}^{\infty} \beta_{k} f_{h_{k}}\left(x-x_{k}\right)\right\|_{C[-1,1]} \leqslant \sum_{k=s+1}^{\infty} \beta_{k}\left\|f_{h_{k}}\right\|_{C[-2,1]} \leqslant \sum_{k=s+1}^{\infty} \beta_{k} \leqslant 2 \beta_{s+1} .
$$

Положим $l:=x_{s-1}, \theta:=x_{s}$. При этом $h_{s} \leqslant c_{2} l^{(q-1) / 2} / n_{s}$ и благодаря (15) имеем $\theta \in(0, l / 3)$. Теперь используя (17)-(19) и А 3$)$, получаем

$$
\begin{aligned}
E_{n_{s}}^{(q)}(f ;[-1,1]) & \geqslant E_{n_{s}}^{(q)}(f ;[-l, l]) \\
& \geqslant E_{n_{s}}^{(q)}\left(\beta_{s} f_{h_{s}}\left(x-x_{s}\right) ;[-l, l]\right)-\left\|\sum_{k=s+1}^{\infty} \beta_{k} f_{h_{k}}\left(x-x_{k}\right)\right\|_{C[-l, l]} \\
& \geqslant \beta_{s} \frac{c_{1} l^{q-1}}{n_{s}^{2}}-2 \beta_{s+1} \geqslant \beta_{s} \frac{c_{1} l^{q-1}}{2 n_{s}^{2}}=\frac{c_{1}}{2 n_{s}^{m+2} n_{s-1}^{(m+1)(q-1)+2}} .
\end{aligned}
$$


Теперь

$$
\alpha_{n_{s}} E_{n_{s}}^{(q)}(f ;[-1,1]) n_{s}^{m+2} \geqslant \frac{c_{1} \alpha_{n_{s}}}{2 n_{s-1}^{(m+1)(q-1)+2}}
$$

и благодаря (12)

$$
\varlimsup_{n \rightarrow \infty} \alpha_{n} E_{n}^{(q)}(f) n^{r-q+3}=+\infty .
$$

Теорема 3 доказана.

Авторы признательны профессору И. А. Шевчуку за внимание к этой работе.

\section{СПИСОК ЦИТИРОВАННОЙ ЛИТЕРАТУРЫ}

[1] Roberts A. W., Varbeg D. E. Convex Functions. New York: Academic Press, 1973.

[2] Lorentz G. G., Zeller K. L. Degree of approximation by monotone polynomials // J. Approximation Theory. 1968. V. 1. № 4. P. 501-504.

[3] Lorentz G. G. Monotone approximation // Inequalities, III / ed. O. Shisha. New York: Academic Press, 1972. P. 201-215.

[4] De Vore R. A. Degree of approximation // Approximation Theory, II / ed. G. G. Lorentz, C. K. Chui, L. Schumaker. New York: Academic Press, 1976. P. 117-162.

[5] Beatson R. K. The degree of monotone approximation // Pacific J. Math. 1978. V. 74. № 1. P. 5-14.

[6] Шведов А.С. Порядки коприближений функций алгебраическими многочленами // Матем. заметки. 1981. Т. 29. №1. С. 117-130.

[7] Шевчук И. А. Приближение многочленами и следы непрерывных на отрезке функций. Киев: Наукова думка, 1992.

[8] Bondarenko A. V. Jackson type inequality in 3-convex approximation // East J. Approx. 2002. V. 8. № 3. P. 291-302.

[9] Konovalov V.N., Leviatan D. Shape-preserving widths of Sobolev-type classes of $s$-monotone functions on a finite interval // Israel J. Math. 2003. V. 133. P. 239-268.

[10] Leviatan D., Shevchuk I. A. Monotone approximation estimates involving the third modulus of smoothness // Approximation Theory IX. Nashville, TN: Vanderbilt University Press, 1998. P. 223-230.

Киевский национальный университет им. Тараса Шевченко, Украина

Поступило

E-mail : bonda@univ.kiev.ua, prymak@univ.kiev.ua

08.05.2003 